\title{
DIFERENCIAÇÃO INTERSETORIAL NA INTERAÇÃO ENTRE EMPRESAS E UNIVERSIDADES NO BRASIL
}

\author{
Eduardo da Motta e Albuquerque \\ Leandro Alves Silva \\ Luciano Póvoa
}

\begin{abstract}
Resumo: Este artigo apresenta resultados preliminares de tabulações especiais preparadas pelo IBGE (a partir da Pintec), focalizando a relação entre universidades e empresas. A hipótese básica é a de que o envolvimento de uma empresa com atividades de P\&D (interna e/ou externa) amplia a importância das universidades como fonte de informação para suas atividades inovativas. Esta hipótese é investigada e se sustenta tanto nos dados gerais como em uma análise intersetorial.

Palavras-chave: Sistemas de inovação. P\&D industrial. Universidades.

Abstract: This short paper presents results based on special tabulations prepared by IBGE, using data from Pintec in order to focus on the interaction between firms and universities in the Brazilian industry. The basic hypothesis states that the relevance of universities as a source of information to the firms' innovation activities is greater when firms are engaged in R\&D activities (both internal and external). The hypothesis is not rejected neither for the industry as a whole nor for inter-sectoral analysis.

Key words: Innovation systems. Industrial R\&D. Universities.
\end{abstract}

$\mathrm{U}$ niversidades e instituições de pesquisa ocupam lugar estratégico nos sistemas nacionais de inovação. Sua infra-estrutura científica relaciona-se com a dimensão tecnológica, colocando-se no centro de uma dinâmica complexa plena de circuitos virtuosos que se reforçam mutuamente. Como Nelson e Rosenberg (1993) colocaram de forma sintética, a ciência tanto lidera como segue o desenvolvimento tecnológico.

No interior da abordagem evolucionista, a partir da elaboração e dos estudos em torno de sistemas nacionais e setoriais de inovação, o tema sobre o papel das universidades vem ganhando importância e atenção. Duas referências recentes são os trabalhos de Mowery et al. (2004) e de Mowery e Sampat (2005). Certamente essa atenção está consoante com a crescente importância das universidades no apoio da capacitação tecnológica de países, regiões, setores e firmas.
Avaliando o Brasil em termos de sua produção científica e tecnológica, é possível classificá-lo como parte de um grupo de países que estariam ainda em processo de construção de seus sistemas de inovação. Assim como México, Índia e África do Sul, o Brasil teria um sistema de inovação caracterizado como imaturo. Nesses países seriam encontradas "conexões parciais" entre a infra-estrutura científica e as atividades tecnológicas (ALBUQUERQUE, 2004). Rapini (2005) apresenta evidências de setores em que se identificariam essas "conexões parciais", indicando aqueles nos quais a interação entre empresas e grupos de pesquisa em universidades já está funcionando efetivamente no Brasil.

A pesquisa que informa este artigo pretende contribuir para a avaliação dessas "conexões parciais" no caso brasileiro. Para mapear essas interações, a Pesquisa Industrial - Inovação Tecnológica 2000 (Pintec), realizada pelo 
IBGE (2002), traz contribuições inestimáveis. A partir de solicitação de um conjunto de tabulações especiais, o IBGE forneceu dados que permitem uma focalização nas relações entre atividade inovativa, P\&D (interno e externo) e importância de universidades e institutos de pesquisa como fonte de informação para as atividades inovativas das empresas. Este artigo apresenta uma avaliação preliminar desses dados.

A hipótese é simples: o envolvimento de uma empresa com atividades de P\&D (interna e/ou externa) amplia a importância das universidades como fonte de informação para suas atividades inovativas. Essa hipótese se relaciona com a identificação já mencionada de "conexões parciais" entre ciência e tecnologia no sistema de inovação brasileiro: essas conexões estariam se estabelecendo a partir de um núcleo de firmas que investem em P\&D.

\section{A UNIVERSIDADE EM UM SISTEMA NACIONAL DE INOVAÇÃO: BREVE REVISÃO DA LITERATURA}

Sistema Nacional de Inovação pode ser definido como um conjunto de instituições, atores e mecanismos de um país que contribuem para criação, avanço e difusão das inovações tecnológicas. Entre estas instituições, atores e mecanismos, destacam-se os institutos de pesquisa, o sistema educacional, as empresas e seus laboratórios de pesquisa e desenvolvimento, a estrutura do sistema financeiro, as leis de propriedade intelectual e as universidades.

A importância do Sistema Nacional de Inovação para o avanço tecnológico não está na existência não apenas deste conjunto de instituições, mas principalmente de fortes interações entre as instituições, permitindo uma atuação conjunta e coerente. Nelson e Rosenberg (1993, p. 11) destacam que as universidades possuem papel importante em um Sistema Nacional de Inovação, atuando como formadoras de cientistas e engenheiros e como fontes de conhecimentos científicos e de pesquisas que fornecem técnicas úteis para o desenvolvimento tecnológico industrial. ${ }^{1}$

Como a estrutura institucional difere entre os países, o papel das universidades, embora importante, varia de intensidade (NELSON, 1988; FREEMAN, 1988) e sua influência pode ser potencializada de acordo com a base industrial de uma região ou país e com a relevância dos incentivos e fundos públicos para a pesquisa científica.

Em um recente trabalho, Mowery e Sampat (2005) apresentam um excelente apanhado dos estudos sobre a importância da pesquisa universitária para os avanços tecnológicos e a interação entre universidade e indústria. Os autores apontam alguns "produtos" economicamente importantes resultantes da pesquisa universitária, tais como: informações tecnológicas e científicas; equipamentos e instrumentação; capital humano; redes de capacidade científica e tecnológica; e protótipos de novos produtos e processos. Destacam também que o fortalecimento da interação entre a universidade e as outras instituições e atores do Sistema Nacional de Inovação, em especial a indústria, é fundamental para que a primeira possa contribuir de forma mais eficaz para o avanço tecnológico.

Vários estudos mostram que tem havido um aumento considerável na cooperação entre universidade e indústria recentemente (MEYER-KRAHMER; SCHMOCH, 1998; COHEN et al., 2002). Este fato deve-se não só ao crescente reconhecimento da importância da pesquisa universitária para as atividades inovativas da indústria, mas também a mudanças estruturais, como restrições orçamentárias relacionadas aos fundos públicos. Desta forma, as universidades têm adotado uma postura mais agressiva e "empresarial" na busca por novas fontes de recursos para a pesquisa (MOWERY; SAMPAT, 2005, p. 211).

Com base no conceito de Sistema Nacional de Inovação, Meyer-Krahmer e Schmoch (1998, p. 847) destacam as seguintes variáveis como determinantes da interação universidade-indústria:

- a "capacidade de absorção" de cada instituição, o que torna possível a interação;

- a estrutura de incentivos das interações, que influencia na intensidade da interação;

- importantes condições macroestruturais (como a característica de centralização do sistema de pesquisa e a orientação de curto ou longo prazo do sistema financeiro) e mesoestruturais (como a estrutura industrial e tecnológica).

É importante destacar que a capacidade de absorção, ou seja, a capacidade da firma em adquirir, da melhor forma possível, os avanços das pesquisas científicas está relacionada aos investimentos internos em P\&D. De acordo com Cohen e Levinthal (1989), os gastos com P\&D não apenas estão ligados ao processo de inovação e aperfeiçoamento de produtos e processos, mas também contribuem para o aprendizado da firma, ou seja, para desenvolver a sua capacidade de absorção.

A contribuição da pesquisa acadêmica para o avanço tecnológico ocorre por vários mecanismos, além de variar entre os setores industriais e de existirem ramos da 
ciência cujos avanços são considerados mais relevantes para as inovações tecnológicas. Klevorick et al. (1995) apontam os avanços no conhecimento científico como sendo a fonte mais importante de oportunidades tecnológicas. ${ }^{2}$ A ciência fornece dados, explicações teóricas, técnicas e soluções gerais de problemas que podem ser utilizadas no desenvolvimento e na pesquisa industrial, além de desenvolver conhecimentos que podem abrir diretamente novas possibilidades tecnológicas (KLEVORICK et al., 1995, p. 193). Através das respostas de questionários enviados às empresas de vários setores, os autores identificaram as indústrias nas quais a relevância da pesquisa universitária para o progresso tecnológico era maior. Os autores também verificaram os ramos da ciência que mais contribuem para os avanços tecnológicos em determinadas indústrias, mostrando que os setores industriais possuem percepções diferenciadas em relação aos avanços de cada campo científico e de pesquisa universitária.

Utilizando o mesmo método de envio de questionários às empresas, Cohen et al. (2002) procuram analisar se a pesquisa pública influencia a geração de novos projetos de P\&D industrial e contribui para a conclusão de projetos existentes. Os resultados sugerem que a pesquisa universitária exerce impacto substancial na pesquisa industrial, mas este impacto é direto em apenas poucas indústrias, como a farmacêutica. Os autores destacam que os principais canais de transmissão de conhecimento das pesquisas públicas para a pesquisa industrial são publicações e relatórios.

\section{O PAPEL DE UNIVERSIDADES NA CONSTRUÇÃO DE SISTEMAS DE INOVAÇÃO NA PERIFERIA}

A situação prevalecente nos países menos desenvolvidos não pode ser compreendida a partir da aplicação direta e sem qualificações das conclusões alcançadas na literatura sobre os países avançados. Há diferenças que devem ser levadas em conta.

No que diz respeito ao papel da ciência, a principal diferença reside na contribuição que ela pode oferecer durante o processo de catching up: a infra-estrutura científica atua como "instrumento de focalização" e "antena" para identificar oportunidades tecnológicas e constituir a capacidade de absorção do país. Em uma nação atrasada, a infra-estrutura científica oferece "conhecimento para focalizar buscas", em vez de ser apenas uma fonte direta de oportunidades tecnológicas. Em outras palavras, a infraestrutura científica em países em desenvolvimento deve contribuir para vincular o país aos fluxos científicos e tecnológicos internacionais.

Neste sentido, o papel da ciência durante processos de catching up pode ser desdobrado em três dimensões: ela atua como "instrumento de focalização", contribuindo para a identificação de oportunidades e para a vinculação do país aos fluxos internacionais; cumpre o papel de instrumento de apoio para o desenvolvimento industrial, provendo conhecimento necessário para a entrada em setores industriais estratégicos (PEREZ; SOETE, 1988); e serve como fonte para algumas soluções criativas que dificilmente seriam obtidas fora do país (exemplo: vacinas contra doenças tropicais, desenvolvimento de certas ligas metálicas, preparação de softwares aplicados, etc.).

Certamente há uma inter-relação entre esses diferentes papéis, uma vez que o desenvolvimento da capacidade de absorção é uma precondição para desenvolvimentos tecnológicos locais, originais e incrementais. ${ }^{3}$

\section{A PINTEC E OS DADOS GERAIS SOBRE EMPRESAS E ATIVIDADES INOVATIVAS}

O principal intuito da Pintec é identificar, de forma rigorosa e pioneira, o envolvimento das empresas brasileiras com atividades inovativas, inclusive identificando o total de gastos em P\&D do setor industrial e o pessoal empregado em atividades de P\&D. ${ }^{4}$ Para uma apreciação geral dos resultados da Pintec, algumas informações são importantes para dimensionar o papel das universidades e instituições de pesquisa:

- a Pintec envolve um total de 72.005 empresas industriais com dez ou mais empregados;

- 22.698 empresas implementaram inovações (de produto e/ou processo), sendo que "aquisição de máquinas e equipamentos" é a atividade inovativa mais importante, com 15.540 empresas informando gastos nesse quesito (IBGE, 2002, p. 52);

- 7.412 empresas informaram gastos com "atividades internas de P\&D”, totalizando R \$ 3,74 bilhões (IBGE, 2002, p. 51);

- nessas 7.412 empresas, foram encontradas 31.447 pessoas ocupadas com "dedicação exclusiva" em atividades de P\&D e 32.945 pessoas ocupadas com "dedicação parcial" (IBGE, 2002, p. 54); 
- avaliando as "fontes de informação empregadas" pelas 22.698 empresas que implementaram inovações, 3.732 informaram utilizar "universidades e institutos de pesquisa" localizados no Brasil e 94 afirmaram que utilizam "universidades e institutos de pesquisa" localizados no exterior (IBGE, 2002, p. 70);

- outra fonte de informação diretamente relacionada à infra-estrutura científica utilizada diz respeito a "conferências, encontros e publicações especializadas", empregada por 8.950 empresas no Brasil e 3.202 no exterior (IBGE, 2002, p. 71);

- quanto às parcerias, 641 empresas consideram importantes as "relações de cooperação" com universidades e institutos de pesquisa.
A partir dessas informações gerais, um conjunto de tabulações especiais foi solicitado ao IBGE, focalizando especificamente as atividades internas de $\mathrm{P} \& \mathrm{D}$ e a importância atribuída pela empresa às universidades e institutos de pesquisa como fonte de informação.

Os dados solicitados envolvem o cruzamento das questões 35 e 36 do questionário da Pintec, respondidas por todos que realizaram atividades internas e/ou externas de P\&D entre 1998 e 2000, e da questão 97, na qual o entrevistado indica o grau de importância (alto, médio, baixo ou não relevante) das universidades e centros de pesquisa como fonte de informação para as atividades inovativas (para esta tabulação especial, o IBGE agregou as respostas em dois grupos: o primeiro com as empresas que responderam importância alta ou média; e o segundo com

\section{QUADRO 1}

Identificação dos Setores

\begin{tabular}{|c|c|}
\hline Código & Nome \\
\hline S1 & Indústrias extrativas \\
\hline S2 & Fabricação de produtos alimentícios e bebidas \\
\hline S3 & Fabricação de produtos de fumo \\
\hline S4 & Fabricação de produtos têxteis \\
\hline S5 & Confecção de artigos do vestuário e acessórios \\
\hline S6 & Preparação de couros e fabricação de artefatos de couro, artigos de viagem e calçados \\
\hline S7 & Fabricação de produtos de madeira \\
\hline S8 & Fabricação de celulose, papel e produtos de papel \\
\hline S9 & Edição, impressão e reprodução de gravações \\
\hline S10 & Fabricação de coque, refino de petróleo, elaboração de combustíveis nucleares e produção de álcool \\
\hline S11 & Fabricação de produtos químicos \\
\hline S12 & Fabricação de artigos de borracha e plástico \\
\hline $\mathrm{S} 13$ & Fabricação de produtos de minerais não-metálicos \\
\hline S14 & Metalurgia básica \\
\hline S15 & Fabricação de produtos de metal \\
\hline S16 & Fabricação de máquinas e equipamentos \\
\hline S17 & Fabricação de máquinas para escritório e equipamentos de informática \\
\hline S18 & Fabricação de máquinas, aparelhos e materiais elétricos \\
\hline S19 & Fabricação de material eletrônico e de aparelhos e equipamentos de comunicações \\
\hline $\mathrm{S} 20$ & $\begin{array}{l}\text { Fabricação de equip. de inst. médico-hospitalares, inst. de precisão e ópticos, equip. para automação } \\
\text { industrial, cronômetros e relógios }\end{array}$ \\
\hline S21 & Fabricação e montagem de veículos automotores, reboques e carrocerias \\
\hline S22 & Fabricação de outros equipamentos de transporte \\
\hline S23 & Fabricação de móveis e indústrias diversas \\
\hline S24 & Reciclagem \\
\hline
\end{tabular}


aquelas que responderam importância baixa ou não relevante).

Além dos dados gerais para o Brasil, solicitou-se ao IBGE a desagregação por setor industrial (classes CNAE, conforme o Quadro 1).

\section{P\&D INDUSTRIAL E IMPORTÂNCIA DAS UNIVERSIDADES}

Firmas que investem em P\&D utilizam mais as universidades como fontes de informação para suas atividades inovativas.

A Tabela 1 apresenta os dados da Pintec indicando a natureza da atividade de P\&D: empresas que realizam apenas P\&D interno; aquelas somente com P\&D externo; as que possuem os dois tipos de P\&D; e aquelas que não desenvolvem P\&D. Assim verifica-se que, entre as empresas com atividades inovativas, $35,6 \%$ investiram em P\&D, sendo 28,2\% apenas em P\&D interno, 2,9\% somente em P\&D externo e 4,5\% nos dois tipos de P\&D.

A relação entre atividades de $P \& D$ e importância da universidade como fonte de informação pode ser identificada comparando os dois conjuntos de firmas: $6,1 \%$ das empresas que não realizam $\mathrm{P} \& \mathrm{D}$ consideram universidades importantes enquanto $21,1 \%$ das que realizaram algum tipo de P\&D utilizaram universidades como fonte de informação (1.701 firmas em 8.062 firmas com P\&D).

Entre as empresas com atividade de P\&D, destaca-se um conjunto minoritário mas relevante: das 1.019 com P\&D interno e externo, 413 (40,6\%) utilizaram universidades e institutos de pesquisa como fontes de informação. Essas firmas devem constituir o núcleo mais dinâmico e capacitado tecnologicamente, que responderia por parte significativa das “conexões parciais” já identificadas no sistema de inovação brasileiro. Possivelmente, há uma relação de complementaridade entre o P\&D das empresas (interno) e o das universidades (externo).

$\mathrm{O}$ conjunto das empresas que realizam apenas $\mathrm{P} \& \mathrm{D}$ externo (649), das quais $222(34,1 \%)$ consideram universidades importantes, ocupa o segundo lugar na valorização das universidades. Possivelmente essas firmas utilizam diretamente as universidades como fonte de seu P\&D (contratação de pesquisas), substituindo (pelo menos temporário) o $\mathrm{P} \& \mathrm{D}$ interno.

O terceiro lugar na valorização das universidades é ocupado pelas empresas que realizam apenas P\&D interno: das 6.394 com atividades internas de P\&D, 1.066 (16,7\%) consideram as universidades importantes. Ressalte-se que essa porcentagem é quase três vezes maior do que a encontrada para as empresas que não realizam P\&D (apenas $6,1 \%$ consideram as universidades importantes).

Finalmente, é necessário um comentário sobre a quantidade de empresas (893) que, embora não realizem P\&D, valorizam as universidades, aproximando-se do total de empresas com P\&D interno que consideram importantes as universidades. Uma explicação para a existência de um número elevado de empresas sem atividades internas de P\&D e atribuindo importância alta e média para as universidades como fonte de informação pode ser a utilização de recursos da universidade como substituto do investimento interno (talvez em função da restrição de recursos financeiros passíveis de alocação para atividades contínuas ou não de P\&D). Outra hipótese seria a de que essas empresas, pelo relacionamento com as universidades, estariam em vias de iniciar atividades internas de P\&D.

TABELA 1

Empresas, por Grau de Importância Atribuído às Universidades como Fonte de Informação, segundo Modalidades de P\&D Brasil - 2000

\begin{tabular}{|c|c|c|c|c|c|c|}
\hline \multirow{2}{*}{ Modalidades de P\&D } & \multicolumn{2}{|c|}{ Alta e Média } & \multicolumn{2}{|c|}{ Baixa e Não-Relevante } & \multicolumn{2}{|c|}{ Total } \\
\hline & №s Absolutos & $\%$ & №s Absolutos & $\%$ & №s Absolutos & $\%$ \\
\hline Total & 2.594 & 11,4 & 20.104 & 88,6 & 22.698 & 100,0 \\
\hline P\&D Interno & 1.066 & 16,7 & 5.327 & 83,3 & 6.394 & 28,2 \\
\hline P\&D Externo & 222 & 34,1 & 427 & 65,9 & 649 & 2,9 \\
\hline P\&D Interno e Externo & 413 & 40,6 & 606 & 59,4 & 1.019 & 4,5 \\
\hline Não Realizam P\&D & 893 & 6,1 & 13.744 & 93,9 & 14.636 & 64,5 \\
\hline
\end{tabular}

Fonte: IBGE. Tabulações especiais. 


\section{DIFERENÇAS INTERSETORIAIS}

\section{Envolvimento em Atividades de P\&D}

O Gráfico 1 sistematiza as diferenças intersetoriais em termos do envolvimento das empresas com atividades de P\&D. Seguindo a divisão apresentada na Tabela 1, os dados são apresentados de acordo com a porcentagem de firmas por setor que realizam P\&D interno, P\&D externo, P\&D interno e externo e as que não realizam P\&D. Os gráficos estão organizados de forma a indicar os setores industriais que apresentam desvio-padrão acima e abaixo das médias setoriais.

Em relação às atividades internas de $\mathrm{P} \& \mathrm{D}$, destacamse os setores 17 (fabricação de máquinas para escritório e equipamentos de informática), 22 (outros equipamentos de transporte), 11 (produtos químicos) e 19 (material ele- trônico e de equipamentos de comunicações), nos quais mais de $50 \%$ das firmas realizam atividades internas de P\&D. No extremo oposto encontram-se os setores 24 (reciclagem), 9 (edição, impressão), 5 (vestuário e acessórios) e 7 (produtos de madeira), com menos de $13 \%$ das empresas realizando P\&D interno.

Quanto às atividades externas de $\mathrm{P} \& \mathrm{D}$, destacam-se os setores 1 (indústrias extrativas), 10 (fabricação de coque, refino de petróleo, nuclear e produção de álcool), 11 (produtos químicos) e 19 (material eletrônico e de equipamentos de comunicações). Nesses setores, de $6 \%$ a $10 \%$ das firmas realizam apenas P\&D externo. No extremo oposto, têm-se os setores 24 (reciclagem), 3 (fumo) e 18 (máquinas e aparelhos elétricos), com menos de $0,2 \%$ das firmas com apenas P\&D externo.

Em relação ao grupo mais sofisticado, que realiza $P \& D$ tanto interno como externo, destacam-se os setores 17 (fa-

\section{GRÁFICO 1}

Proporção das Firmas, por Setor, segundo Modalidade de P\&D - Média e Desvio-Padrão

Brasil - 2000
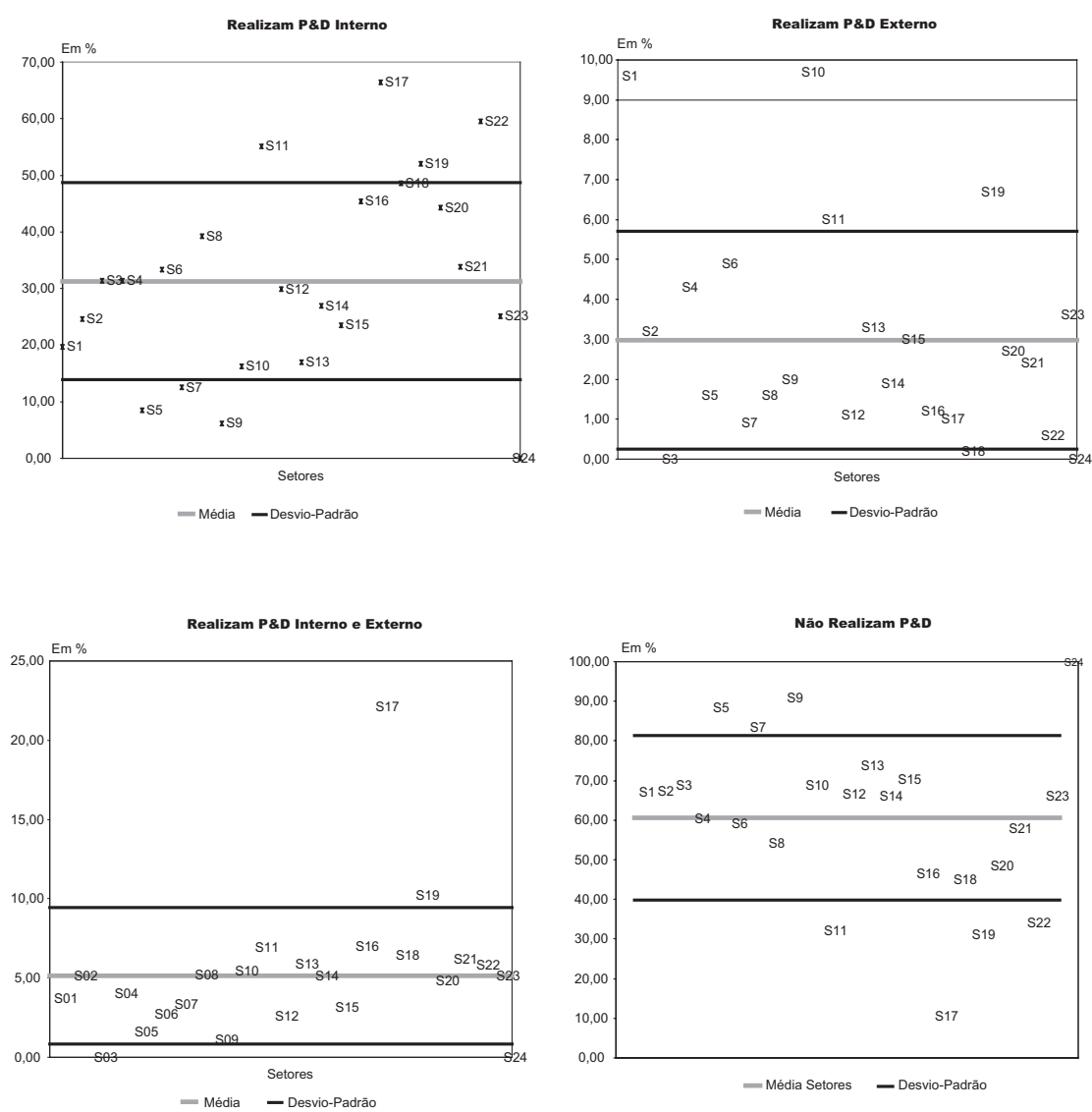

Fonte: IBGE. Tabulações especiais.

Nota: Para a identificação dos setores, consulte o Quadro 1. 
bricação de máquinas para escritório e equipamentos de informática) e 19 (material eletrônico e de equipamentos de comunicações), com mais de $10 \%$ das empresas com tais atividades. No extremo oposto, novamente aparecem os setores 3 (fumo) e 24 (reciclagem), sem nenhuma firma envolvida.

Finalmente, o Gráfico 1 apresenta os setores que se destacam pela não realização de P\&D: com mais de $80 \%$ das empresas sem estas atividades, encontram-se os setores 24 (reciclagem), 9 (edição, impressão), 5 (vestuário e acessórios) e 7 (produtos de madeira). No extremo oposto, com menos de $35 \%$ das firmas não envolvidas, estão os setores 17 (fabricação de máquinas para escritório e equipamentos de informática), 19 (material eletrônico e de equipamentos de comunicações), 11 (produtos quími$\cos$ ) e 22 (outros equipamentos de transporte).

\section{P\&D e Importância de Universidades}

O Gráfico 2 permite avaliar a correlação entre o nãoenvolvimento com atividades de P\&D e a importância das universidades para a inovação no setor. O resultado mais importante refere-se à relação inversa entre o nãoenvolvimento de um setor industrial com atividades de
P\&D e a tendência de valorizar as universidades como fonte de informação. À medida que diminui a média setorial de "não-envolvimento" com P\&D, cresce a importância das universidades para as atividades inovativas.

O Gráfico 2 mostra, em um extremo, o setor 24 (reciclagem), com $100 \%$ das firmas não-envolvidas com P\&D e $0 \%$ considerando universidades importantes e, no outro, se o setor 17 (fabricação de máquinas para escritório e equipamentos de informática), com cerca de $10 \%$ das firmas não-envolvidas com P\&D e com cerca de $30 \%$ atribuindo importância às universidades.

O setor 20 (equipamentos médico-hospitalares, instrumentos de precisão, equipamentos de automação industrial) destaca-se como o que mais valoriza as universidades (um pouco mais de 30\% das empresas), embora esteja em sétimo lugar em termos de envolvimento com P\&D. É também importante mencionar o setor 14 (metalurgia básica), que, embora esteja em décimo terceiro lugar em termos de envolvimento com P\&D, é o quarto setor que mais valoriza as universidades. Isso talvez expresse a tradição do setor e o envolvimento histórico com universidades. Aliás, o quinto lugar em termos da valorização de universidades encontra-se com as indústrias extrativas (S1), que detêm apenas a décima sexta colocação em termos de

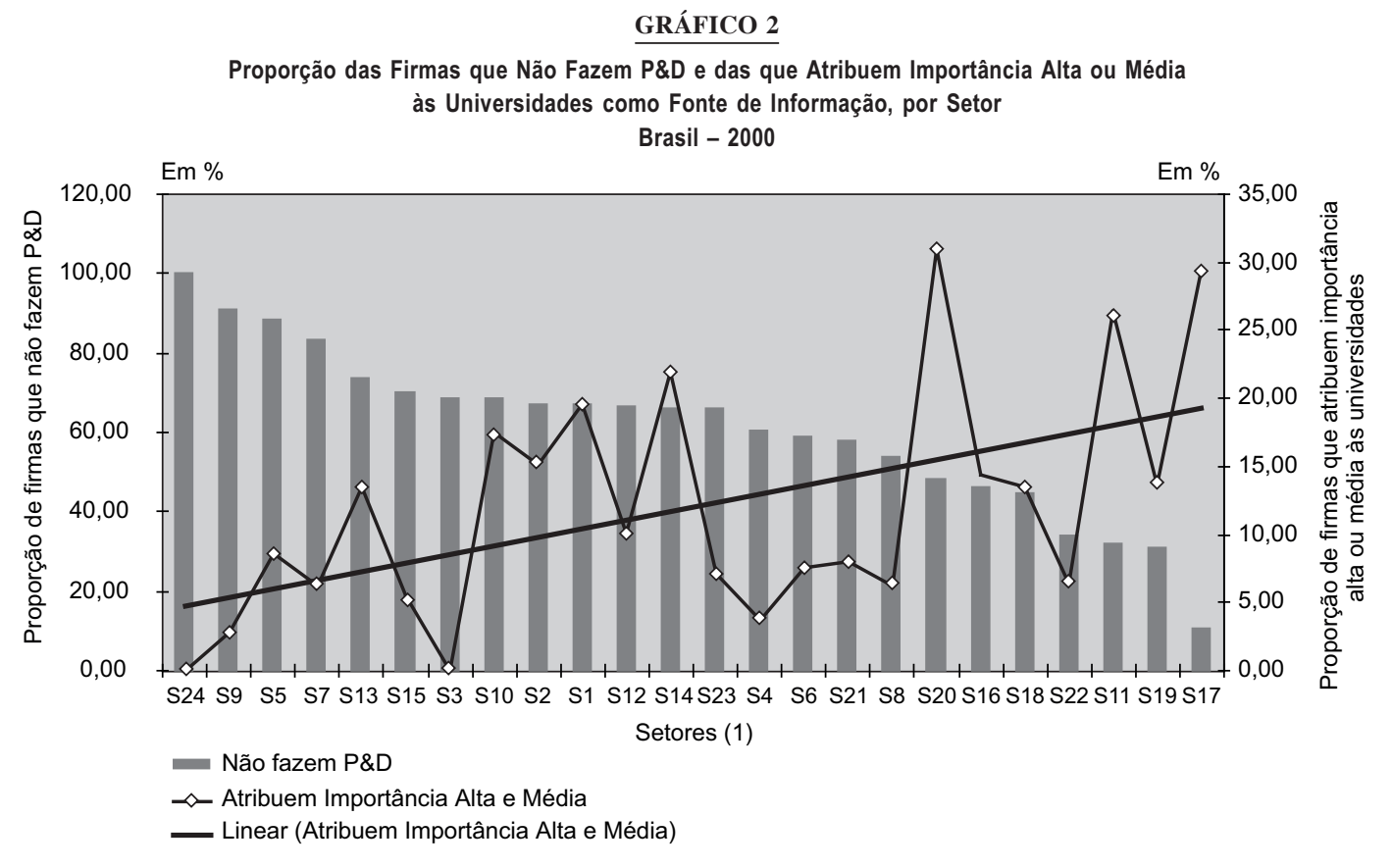

Fonte: IBGE. Tabulações especiais.

(1) Para identificação dos setores, ver Quadro 1 
envolvimento com P\&D. Esses dois comentários podem indicar que a base para a competitividade nesses setores importantes na economia brasileira (que não são de alta tecnologia) depende das atividades de universidades.

\section{P\&D Interno e Externo e Universidades}

O Gráfico 3 focaliza o que pode ser considerado o grupo mais sofisticado: as empresas que realizam simultaneamente $\mathrm{P} \& \mathrm{D}$ interno e externo. O resultado mais importante indicado é a relação direta entre um setor industrial que desenvolve atividades internas e externas de P\&D e a tendência de valorizar as universidades como fonte de informação. À medida que decresce a média setorial do envolvimento com P\&D interno e externo, diminui a tendência de valorizar as universidades como fonte de informação para as atividades inovativas.

Os setores localizados nos extremos do Gráfico 3 são os mesmos do Gráfico 2, mas com posições invertidas: de um lado está o setor 17 (fabricação de máquinas para escritório e equipamentos de informática), com mais de $20 \%$ das firmas com atividades internas e externas de $\mathrm{P} \& \mathrm{D}$, das quais cerca de $20 \%$ valorizam as universidades como fonte de informação; e, do outro, o setor 24 (reciclagem), com
$0 \%$ das empresas envolvidas com P\&D interno e externo e com $0 \%$ considerando universidades importantes.

Destaca-se que, em décimo segundo lugar entre os setores com mais envolvimento simultâneo em P\&D interno e externo, está o setor que mais valoriza as universidades: o 14 (metalurgia básica), com cerca de 5\% das empresas realizando $\mathrm{P} \& \mathrm{D}$ interno e externo, das quais mais de $80 \%$ consideram as universidades importantes. É interessante também notar que com mais de $65 \%$ das empresas com P\&D interno e externo valorizando as universidades estão os setores 10 (fabricação de coque, refino de petróleo, nuclear e produção de álcool), 11 (produtos químicos) e 1 (indústrias extrativas).

Esses comentários reforçam novamente a indicação de que a base para a competitividade, nesses setores importantes da economia brasileira (com exceção do setor 11 , produtos químicos, não são de alta tecnologia), parece depender, de alguma forma, da interação desses setores com atividades de universidades e institutos de pesquisa.

\section{COMENTÁRIOS FINAIS}

A hipótese básica deste artigo (o envolvimento de uma empresa com atividades de P\&D amplia a importância das

\section{GRÁFICO 3}

Proporção das Firmas com P\&D Interno e Externo e das que Atribuem Importância Alta ou Média às Universidades como Fonte de Informação, por Setor Brasil - 2000

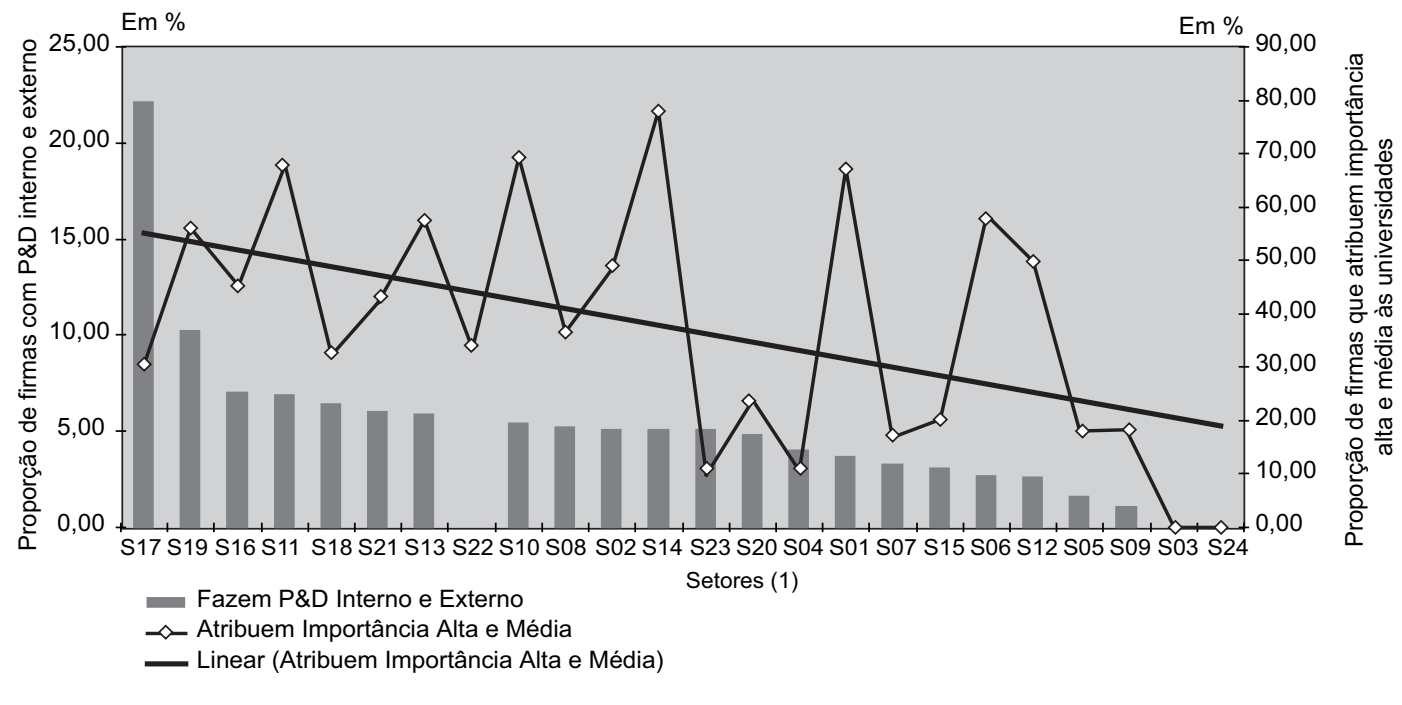

Fonte: IBGE. Tabulações especiais.

(1) Para identificação dos setores, ver Quadro 1. 
universidades como fonte de informação para suas atividades inovativas) não foi refutada pelos dados apresentados. Além disso, essa relação entre investimentos em P\&D utilização de universidades e institutos de pesquisa como fontes de informação sustenta-se em comparações intersetoriais.

Os dados apresentados sugerem que as empresas ao realizarem investimentos em $\mathrm{P} \& \mathrm{D}$, ampliam sua capacidade de absorção de conhecimentos e, por isso, tendem a valorizar mais as universidades como fonte de informação.

Essa relação pode ser mais investigada por meio de estudos de caso e de novas avaliações quantitativas a partir dos dados da Pintec.

Há um duplo papel para as universidades na discussão aqui realizada: por um lado, a ampliação de investimentos em P\&D multiplica a importância das universidades como fonte de informação para atividades inovativas empresariais (os canais dessas fontes de informação são diversos: publicações, contratação de pessoal, participação em congressos, patentes, contratos, pesquisa conjunta, etc.); e, por outro, o início e a ampliação de investimentos em $\mathrm{P} \& \mathrm{D}$ dependem de profissionais com formação universitária atuando nas empresas. Essa dupla função possivelmente sugere que o papel das universidades e institutos de pesquisa nos processos de catching up contemporâneos é maior do que o normalmente considerado.

\section{NOTAS}

Os dados aqui apresentados foram fornecidos pelo IBGE, em tabulações especiais. As contribuições de Mariana Rebouças e do Deind do IBGE são essenciais para a implementação da pesquisa que informa esta comunicação. A preparação deste texto contou com a colaboração das bolsistas de iniciação científica Elaine Rodrigues, Thaís Henriques e Raquel Guimarães. Esta pesquisa é apoiada pelo CNPq e pelo NepaqUFMG.

1. Narin et al. (1997) fornecem evidências empíricas da crescente influência das pesquisas universitárias e de instituições de pesquisa governamentais como fonte de conhecimento para as inovações tecnológicas nos Estados Unidos.

2. As oportunidades tecnológicas compreendem o "conjunto de possibilidades para o avanço tecnológico" (KLEVORICK et al., 1995, p. 188).

3. Para uma discussão mais geral das relações entre processos de catching up e tecnologia, ver Albuquerque (1997).

4. Para uma discussão detalhada da construção da Pintec, ver Bastos, Rebouças e Bivar (2003).

\section{REFERÊNCIAS BIBLIOGRÁFICAS}

ALBUQUERQUE, E. Notas sobre os determinantes tecnológicos do catching up: uma introdução à discussão sobre o papel dos sistemas nacionais de inovação na periferia. Estudos econômicos, v. 27, n. 2, p. 221-253, 1997.

ALBUQUERQUE, E. Science and technology systems in Less Developed countries: identifying a threshold level and focusing in the cases of India and Brazil. In: MOED, H.; GLÄNZEL, W.; SCHMOCH, U. (Ed.). Handbook of quantitative science and technology research: the use of publication and patent statistics in studies of S\&T systems. Dordrecht: Kluwer Academic Publishers, 2004.

BASTOS, C.; REBOUÇAS, M.; BIVAR, W. A construção da Pesquisa Industrial de Inovação Tecnológica - Pintec. In: VIOTTI, E.; MACEDO, M.M. (Org.). Indicadores de ciência, tecnologia e inovação no Brasil. Campinas: Editora Unicamp, 2003. p. 463-532.

COHEN, W.; LEVINTHAL, D. Innovation and learning: the two faces of R\&D. The Economic Journal, v. 99, n. 397, p. 569-596, 1989.

COHEN, W.; NELSON, R.; WALSH, J. Links and impacts: the influence of public R\&D on industrial research. Management Science, v. 48, n. 1, p. 1-23, 2002.

COLYVAS, J.; CROW, M.; GELIJNS, A.; MAZZOLENI, R.; NELSON, R.; ROSENBERG, N.; SAMPAT, B. How do university inventions get into pratice? Management Science, v. 48, n. 1, p. 61-72, 2002.

FREEMAN, C. Japan: a new National System of Innovation? In: DOSI, G.; FREEMAN, C.; NELSON, R.; SILVERBERG, G.; SOETE, L. (Ed.). Technical change an economic theory. London and New York, Printer Publishers, 1988. p. 330-348.

IBGE. Pesquisa Industrial - Inovação Tecnológica - Pintec 2000. Rio de Janeiro: 2002.

KLEVORICK, A.; LEVIN, R.; NELSON, R.; WINTER, S. On the sources and significance of inter-industry differences in technological opportunities. Research Policy, v. 24, p. 185-205, 1995.

MEYER-KRAHMER, F.; SCHMOCH, U. Science-based technologies: industry-university interactions in four fields. Research Policy, v. 27, p. 835-851, 1998.

MOWERY, D.; NELSON, R.; SAMPAT, B.; ZIEDONIS, A. Ivory tower and industrial innovation: university-industry technology transfer before and after the Bayh-Dole Act. Stanford: Stanford University, 2004.

MOWERY, D.; SAMPAT, B. Universities in national innovation systems. In: FARGERBERG, J.; MOWERY, D.; NELSON, R. (Ed.). The Oxford Handbook of Innovation. Oxford University Press, p. 209-239, 2005.

NARIN, F.; HAMILTON, K.S.; OLIVASTRO, D. The increasing linkage between U.S. technology and public science. Research Policy, v. 26, n. 3, p. 317-330, 1997.

NELSON, R. Institutions supporting technical change in the United States. In: DOSI, G.; FREEMAN, C.; NELSON, R.; SILVERBERG, G.; SOETE, L. (Ed.). Technical change an economic theory. London and New York: Printer Publishers, 1988. p. 312-329. 
NELSON, R.; ROSENBERG, N. Technical innovation and national systems. In: NELSON, R. (Ed.). National innovation systems: a comparative analysis. New York: Oxford University, 1993. p. 3-21.

PAVITT, K. What makes basic research economically useful? Research Policy, v. 20, n. 2, p. 109-119, 1991.

PEREZ, C.; SOETE, L. Catching up in technology: entry barriers and windows of opportunity. In: DOSI, G.; FREEMAN, C.;

NELSON, R. et al. (Ed.). Technical change and economic theory. London and New York: Pinter Publishers, 1988. p. 458-479.

RAPINI, M.S. Interação universidade-empresa no Brasil: evidências do diretório dos grupos de pesquisa do CNPq. Belo Horizonte: UFMG/Cedeplar, 2005. 24p. (Texto para Discussão, 251). Disponível em: 〈http://www.cedeplar.ufmg.br/pesquisas/td/TD 251.pdf>.

ROSENBERG, N. Schumpeter and the endogeneity of technology: some American perspectives. London: Routledge, 2000 .
Inside the black box: technology and economics. Cambridge: Cambridge University, 1982

Eduardo da Motta e Albuquerque: Professor Adjunto da Faculdade de Ciências Econômicas da Universidade Federal de Minas Gerais.

Leandro Alves Silva: Mestre em Economia, Pesquisador do Centro de Desenvolvimento e Planejamento Regional da Faculdade de Ciências Econômicas da UFMG.

Luciano Póvoa: Mestre em Economia.

Artigo recebido em 24 de março de 2005. Aprovado em 13 de abril de 2005. 